OPEN ACCESS

Edited by:

Andreas Recke,

University of Lübeck, Germany

Reviewed by:

Chunpeng Wu

Global Energy Interconnection

Research Institute, China

Hsinyu Tsai,

IBM Research, United States

*Correspondence:

Yu Fu

baoyingqiu0806@126.com

tThese authors have contributed equally to this work

Specialty section:

This article was submitted to Dermatology,

a section of the journa

Frontiers in Medicine

Received: 16 April 2021 Accepted: 24 June 2021

Published: 16 July 2021

Citation:

Bao Y, Zhang J, Zhang Q, Chang J,

Lu D and Fu Y (2021) Artificial

Intelligence-Aided Recognition of

Pathological Characteristics and Subtype Classification of Superficial

Perivascular Dermatitis.

Front. Med. 8:696305

doi: 10.3389/fmed.2021.696305

\section{Artificial Intelligence-Aided Recognition of Pathological Characteristics and Subtype Classification of Superficial Perivascular Dermatitis}

\author{
Yingqiu Bao ${ }^{1 \dagger}$, Jing Zhang ${ }^{2,3+}$, Qiuli Zhang ${ }^{1}$, Jianmin Chang ${ }^{1}$, Di Lu ${ }^{3}$ and Yu Fu ${ }^{1 *}$ \\ ${ }^{1}$ Department of Dermatology, Beijing Hospital, National Center of Gerontology, Beijing, China, ${ }^{2}$ Department of Biomedical \\ Engineering, Tsinghua University, Beijing, China, ${ }^{3}$ Bodhi Lab., Beijing BeYes Technology Co. Ltd., Beijing, China
}

Background: Superficial perivascular dermatitis, an important type of inflammatory dermatosis, comprises various skin diseases, which are difficult to distinguish by clinical manifestations and need pathological imaging observation. Coupled with its complex pathological characteristics, the subtype classification depends to a great extent on dermatopathologists. There is an urgent need to develop an efficient approach to recognize the pathological characteristics and classify the subtypes of superficial perivascular dermatitis.

Methods: 3,954 pathological images $(4 \times$ and $10 x$ ) of three subtypes-psoriasiform, spongiotic and interface-of superficial perivascular dermatitis were captured from 327 cases diagnosed both clinically and pathologically. The control group comprised 1,337 pathological images of 85 normal skin tissue slides taken from the edge of benign epidermal cysts. First, senior dermatologists and dermatopathologists followed the structure-pattern analysis method to label the pathological characteristics that significantly contribute to classifying different subtypes on $4 x$ and $10 x$ images. A cascaded deep learning algorithm framework was then proposed to establish pixel-level pathological characteristics' masks and classify the subtypes by supervised learning.

Results: 13 different pathological characteristics were recognized, and the accuracy of subtype classification was $85.24 \%$. In contrast, the accuracy of the subtype classification model without recognition was $71.35 \%$.

Conclusion: Our cascaded deep learning model used small samples to deliver efficient recognition of pathological characteristics and subtype classification simultaneously. Moreover, the proposed method could be applied to both microscopic images and digital scanned images.

Keywords: superficial perivascular dermatitis, skin histopathology images, multitask deep learning, pathological characteristics, subtype classification 


\section{INTRODUCTION}

Inflammatory dermatoses constitute a major category of skin diseases, including many common conditions such as psoriasis, lichen planus, and eczema. For cases with atypical clinical manifestation, a pathological imaging observation can distinguish inflammation subtypes based on pathological characteristics and thus provide key clues for dermatologists to formulate diagnoses and treatments. Professor A. Bernard Ackerman first proposed the structure-pattern analysis method in the 1960s, which laid the foundation for dermatopathology (1). Among the inflammatory dermatoses, superficial perivascular dermatitis is an important pathological type with four subtypes: simplex, interface, psoriasiform and spongiotic (2). However, identification of the pathological characteristics and subtypes of inflammation remains time consuming and relies on professional experience. Furthermore, the lack of senior dermatopathologists in China makes the task more difficult. Therefore, developing an efficient approach to recognize pathological characteristics and classify subtypes of inflammation is essential.

Recently, some strategies based on artificial intelligence (AI) and machine learning, including deep learning networks, have been developed and are being widely used in dermatopathology. Hekler et al. (3) established a deep learning classification model using 695 whole-slide images to distinguish melanoma or benign nevus, achieving an accuracy of $68 \%$. In comparison, the average accuracy of 11 other dermatologists was 59.2\%. Jiang et al. (4) established a deep learning segmentation model, based on 8,046 pathological images collected using a smartphone and achieved automatic recognition and segmentation of basal cell carcinoma. The model reached an intersection over union (IoU) of 0.863 , a specificity of 0.969 , and a sensitivity of 0.939 , respectively, which were significantly better than the previous IoU (0.764) (5). specificity (0.927), and sensitivity (0.869) (6). The above studies show that deep learning models can be used to classify pathological images and recognize pathological characteristics.

Current studies are focused on skin tumors, and there has been no related study on artificial intelligence-aided recognition of pathological characteristics and classification of skin inflammation. In this study, following the structurepattern analysis method, a cascaded deep learning framework was established in combination with a pixel-level segmentation model to achieve pathological characteristics' masks and a model to classify the subtypes of superficial perivascular dermatitis. It would be an efficient approach to recognize pathological characteristics and classify subtypes of superficial perivascular dermatitis simultaneously.

The incidence of inflammatory dermatoses, including many common conditions such as psoriasis, eczema, and lichen planus, is high in China. Because some inflammatory dermatoses with atypical manifestations are difficult to distinguish, histopathology images play an important role in helping clinicians make the right decision. Ackerman's structure-pattern analysis is a useful means for identifying the pathological subtypes of inflammatory skin diseases (7), and superficial perivascular dermatitis is the most common inflammatory skin disease. However, given the complex pathological patterns of inflammatory skin diseases and the lack of senior dermatopathologists, pathological identification remains time consuming and depends on professional experience. In recent years, the progress of AI technology in the medical field is particularly reflected in the rapid recognition and classification of various medical images. Computer vision and deep learning methods are being increasingly applied in dermatology and dermatopathology, and dermatologists are generally positive about the increased use of AI. Among 718 physicians from 91 countries, $72.3 \%$ agreed or strongly agreed that AI would improve dermatopathology (8). In 2017, a Stanford team used deep learning to automatically classify nearly 130,000 dermoscopic images of clinical skin lesions (9). They employed the "GoogleNet Inception-V3" classification framework, used transfer learning to fine-tune model parameters, and obtained the final convolutional neural network model. Using the model, the team completed the diagnostic tasks of melanoma and benign pigmented moles and achieved a recognition result equivalent to that of a dermatologist. Olsen et al. (10) established a deep learning classification model using 450 whole-slide images to classify basal cell carcinoma, dermal nevus, and seborrheic keratosis, obtaining the area under the curve results of $0.99,0.97$, and 0.99 , respectively. Achi et al. (11) established a deep learning classification model using 128 wholeslide images and achieved an image-based accuracy of $95 \%$ and a test set-based accuracy of $100 \%$ in classifying four types of lymphomas: benign lymph node, diffuse large B-cell lymphoma, Burkitt lymphoma, and small lymphocytic lymphoma. Current studies primarily focus on recognition of dermoscopic images and pathological recognition of benign/malignant lesions. There was no relevant research on the recognition of pathological characteristics and classification of skin inflammation (12). This study was the first to use AI technology to assist in pathological recognition and classification of inflammatory dermatosis.

The challenges of recognizing mainly focus on low contrast and having cluttering background in the process of pathological characteristics' recognition. Moreover, the whole-slide is high resolution. To solve the above, this study uses pipeline processing for pathological characteristics' recognition and subtype classification. First, the pathological characteristics are recognized by segmentaion model at $4 \times$ and $10 \times$ separately. The pathological characteristics are typically recognizable under the current magnification and contribute to the subtype classification, so as to reduce the influence of low contrast and cluttering background as much as possible. In addition, the $4 \times$ images and $10 \times$ images belonging to the same slide are used as a set to simulate the high-resolution mode of the wholeslide for classification. For the model selection, the strategy is to use better-performing segmentation algorithms and classification algorithms. Finally we choose deeplabV3 + from deeplabV1, $\mathrm{V} 2, \mathrm{~V} 3, \mathrm{~V} 3+$, which is more sensitive to different scales, and EfficientNet-B1 which can carry out multi-channel input.

\section{MATERIALS AND METHODS}

\section{Dataset}

This was a retrospective study and the samples were obtained from the pathological slides that have been preserved at the 
TABLE 1 | The details of superficial perivascular dermatitis dataset.

\begin{tabular}{|c|c|c|c|c|c|}
\hline Magnifications & Psoriasiform & Spongiotic & Interface & Normal & Total \\
\hline & Train/Test images & Train/Test Images & Train/Test images & Train/Test images & Train/Test images \\
\hline $4 \times$ & $564 / 44$ & $507 / 38$ & $396 / 50$ & $507 / 44$ & $1974 / 176$ \\
\hline $10 x$ & $772 / 58$ & $747 / 73$ & $592 / 113$ & $714 / 72$ & $2825 / 316$ \\
\hline
\end{tabular}

Dermatology Department of Beijing Hospital from Jan 2015 to Jun 2020. Senior dermatologists and dermal pathologists selected pathological slides that had been diagnosed both clinically and pathologically, and the most representative diseases of the three subtypes of superficial perivascular dermatitis-psoriasiform, spongiotic and interface-were chosen, including psoriasis, acute eczema and contact dermatitis, lichen planus, and lichen sclerosus et atrophicus. In total, the histopathology slides of 412 cases were selected to collect 3,954 images from 327 cases of three subtypes and 1,337 images from 85 cases of normal skin histopathology slides taken from the edge of benign epidermal cysts. There were 5,291 images in total, including $4 \times$ images and $10 \times$ images. The details of the dataset are shown in Table 1. One subtype classification sample set contained two $4 \times$ images and two $10 \times$ images of the same slide. In addition, 2,435 images were captured from microscope screenshots and the other 2,856 images were captured from digitally scanned whole-slide images.

All images were labeled manually by senior dermatologists and dermatopathologists. This study was approved by the Ethics Committee of Beijing hospital.

\section{Labeling of Pathological Characteristics}

The principle of structure-pattern analysis method is observing the pathological characteristics ranging from low magnification to high magnification and then identifying the subtypes of superficial perivascular dermatitis, which was based on the labeling of pathological characteristics in this study. Rather than labeling all pathological changes, the pathological characteristics were labeled by considering their contribution to subtype classification, which could help to reduce the deviation in identification between physicians. There are two principles for selecting pathological characteristics in this study. One is that the characteristic is clearly visible at the current magnification, and then it is judged by experienced dermatopathologists that this characteristic has a significant contribution to the subtype of inflammation. For example, hyperkeratosis, acanthosis and infiltration of perivascular inflammatory cells are more obvious at $4 \times$ than $10 \times$, which are the typical characteristics of psoriasiform subtype at $4 \times$. The labeling regions of the three subtypes are shown in Figure $\mathbf{1}$ and Table 2. The labeling software was labelme.exe compiled by Beyes Tech.

\section{Cascaded Deep Learning Framework}

The overall framework of this model was composed of preprocessing and cascaded recognition model for pathological characteristics and subtype classification, shown in Figure 2. After labeling, each sample was normalized (preprocessing) and treated using the deep learning models for segmentation and classification, thus obtaining its pathological characteristics' mask and subtype of superficial perivascular dermatitis.

\section{Normalization (Preprocessing)}

Microscopic images were normalized via matting of the foreground and background, color space normalization, and cropping of digitally scanned images to $1,024 \times 1,024$, as shown in Figure 3. In order to unify the color of the microscope images to the digital scanned images, the algorithm used in this study is background matting, which is the trimap-free automatic matting algorithm that utilizes a casually captured background (13). Thus, microscopic images and digitally scanned images were made consistent in color space and size to reduce differences and facilitate uniform recognition of pathological characteristics and subtype classification modeling.

\section{Modeling of Recognition of Pathological Characteristics}

The sampled $4 \times$ and $10 \times$ images were used for recognition of pathological characteristics. The deep learning segmentation model "deeplabV3+" was used for supervised learning. The region masks generated by $4 \times$ images were hyperkeratosis, acanthosis, inflammatory cell infiltration, and lichenoid infiltration. The region masks generated by $10 \times$ images were parakeratosis, spongiosis, melanophages, hypergranulosis, hypogranulosis, blister, liquefaction degeneration of basal cells, angiectasis of dermal papillae, and thinning of the suprapapillary epidermis. The DeepLabV3+ parameter settings of training: $\mathrm{LR}=0.01$, LR_GAMMA $=0.1$, BATCHES $=4$,EPOCHS $=500$.

\section{Modeling of Subtype Classification}

The deep learning classification model "EfficientNet-B1" was used for supervised learning. The input was 4 images' channles, followed by $4 \times$ original image and its pathological characteristics' mask, $10 \times$ original image and its pathological characteristics' mask. The output was four subtypes: psoriasiform, spongiotic, interface, and normal, as shown in Figure 4. The original images were randomly increased with Gaussian noise and salt-andpepper noise to avoid overfitting of the training set. The adding method was $45 \%$ Gaussian noise and $45 \%$ salt-and-pepper noise. The Efficientnet-B1 parameter settings of training: LR $=1 \mathrm{e}-4$, batch_size $=8, \mathrm{EPOCH}=201$, betas $=(0.9,0.999)$, eps $=1 \mathrm{e}-9$.

Finally, the performance of our model is evaluated by accuracy, sensitivity, and specificity. The formula for sensitivity 


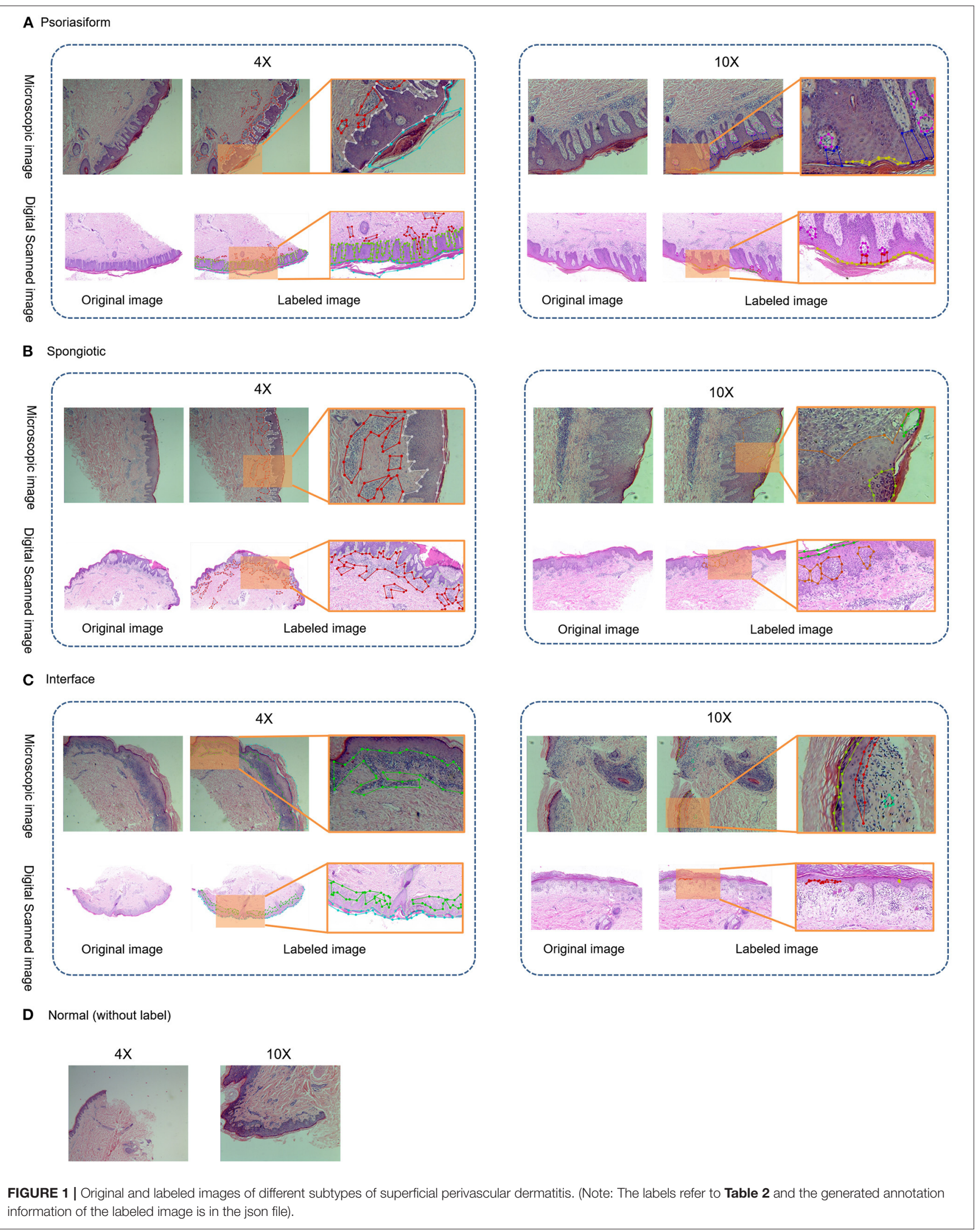


TABLE 2 | Labels and labeling magnifications.

\begin{tabular}{|c|c|c|c|}
\hline Magnifications & Psoriasiform & Interface & Spongiotic \\
\hline $4 \times$ & $\begin{array}{l}\text { Hyperkeratosis, acanthosis, infiltration of } \\
\text { perivascular inflammatory cells }\end{array}$ & $\begin{array}{l}\text { Hyperkeratosis, acanthosis, lichenoid } \\
\text { infiltration of inflammatory cells }\end{array}$ & $\begin{array}{l}\text { Hyperkeratosis, acanthosis, infiltration } \\
\text { of perivascular inflammatory cells }\end{array}$ \\
\hline $10 x$ & $\begin{array}{l}\text { Parakeratosis, hypogranulosis, angiectasis } \\
\text { of dermal papillae and thinning of the }\end{array}$ & $\begin{array}{l}\text { Melanophage, hypergranulosis, liquefaction } \\
\text { degeneration of basal cells }\end{array}$ & $\begin{array}{l}\text { Parakeratosis, spongiosis, } \\
\text { hypergranulosis, blister }\end{array}$ \\
\hline
\end{tabular}

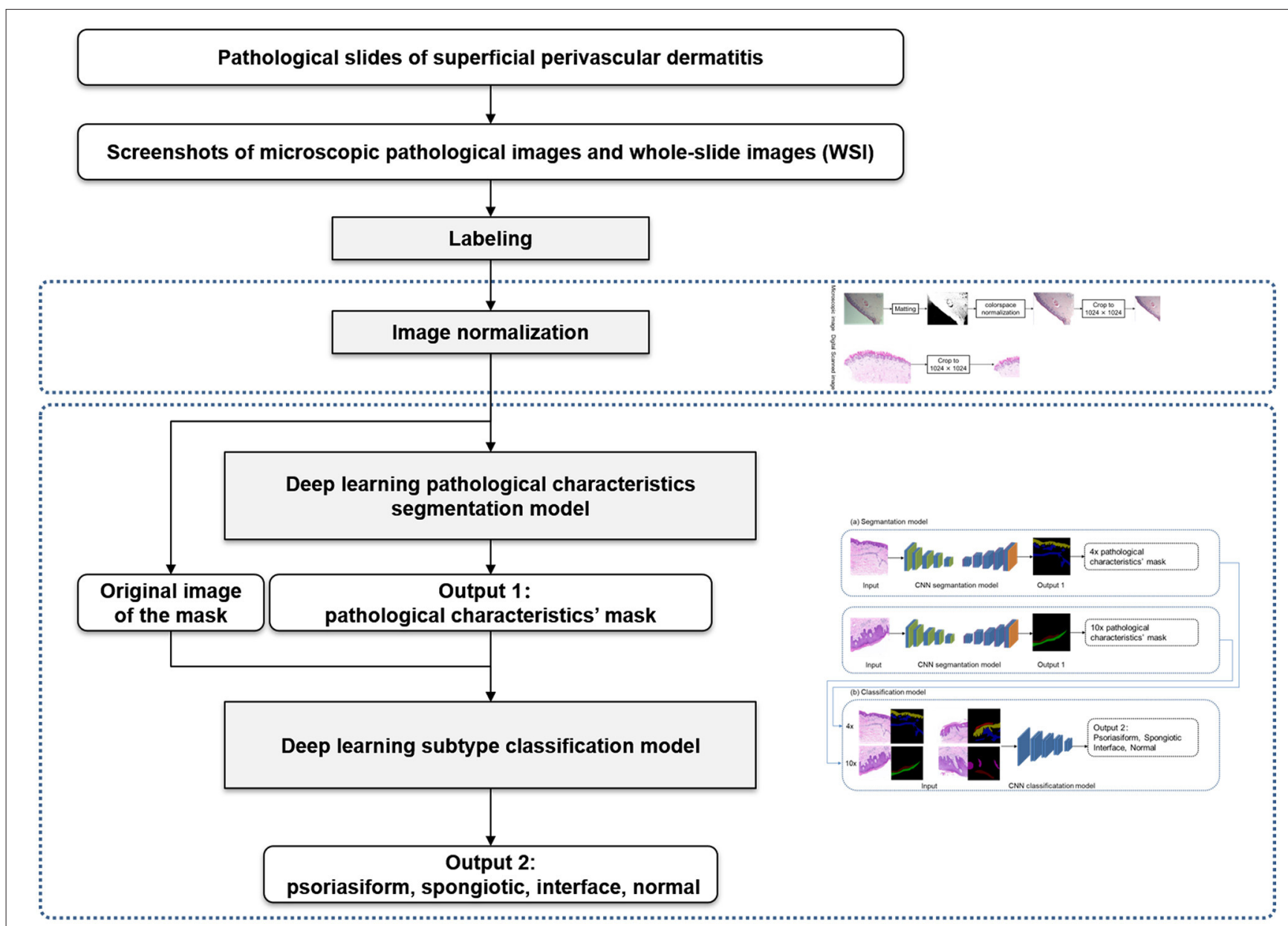

FIGURE 2 | Overall framework of the model.

and specificity is:

$$
\text { sensitivity }=T P / P
$$

TP, The number of sets that are actually positive and classified as positive; $\mathrm{P}$, The number of the sets that are actually positive.

$$
\text { specificity }=T N / N
$$

TN, The number of sets that are actually negative and classified as negative; $\mathrm{N}$, The number of the sets that are actually negative.

\section{RESULTS}

\section{Modeling Results and Evaluation}

Our subtype classification accuracy of the four types in the test set was $85.24 \%$, and the sensitivity and the specificity were 67.46 and $89.09 \%$, respectively. In contrast, the accuracy of Efficientnet-B1 subtype classification without recognition was $71.35 \%$; the accuracy of Efficientnet-B1 cascaded UNet was 79.88\%; the accuracy of ResNet152 cascaded was $80.76 \%$. Table 3 shows the subtype classification results, and Table 4 shows the different model parameter settings of training. The accuracy of each subtype of our model is 99.79\%(normal), $81.12 \%$ (pongiotic), $83.69 \%$ (interface), and 


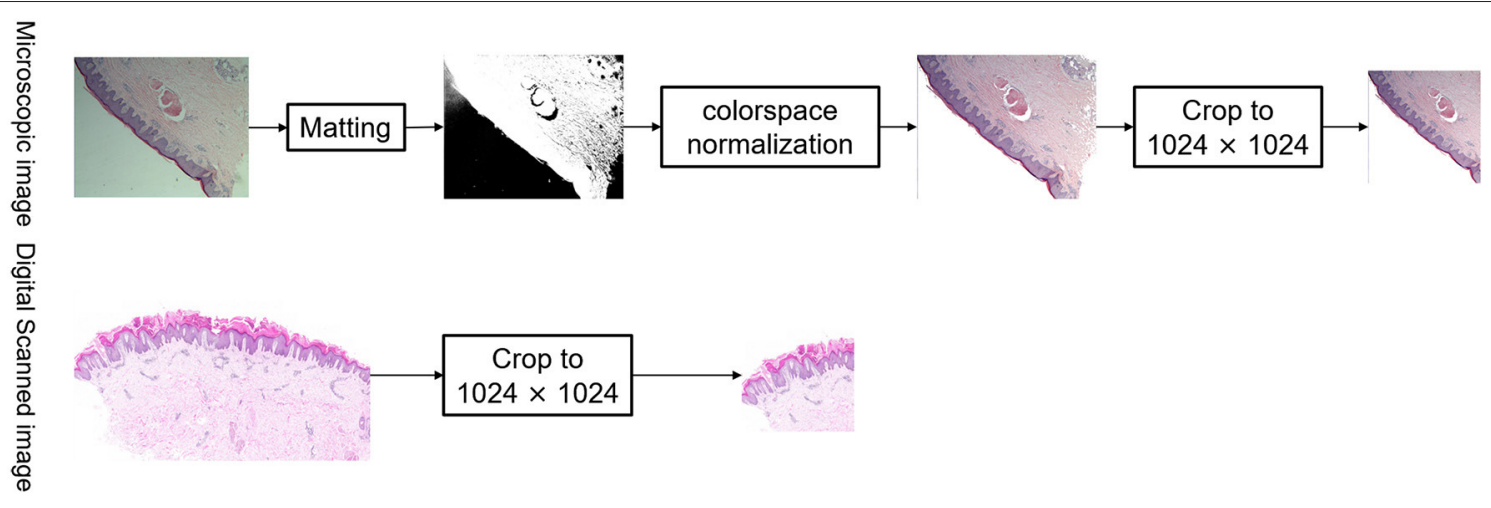

FIGURE 3 | Normalization of images.

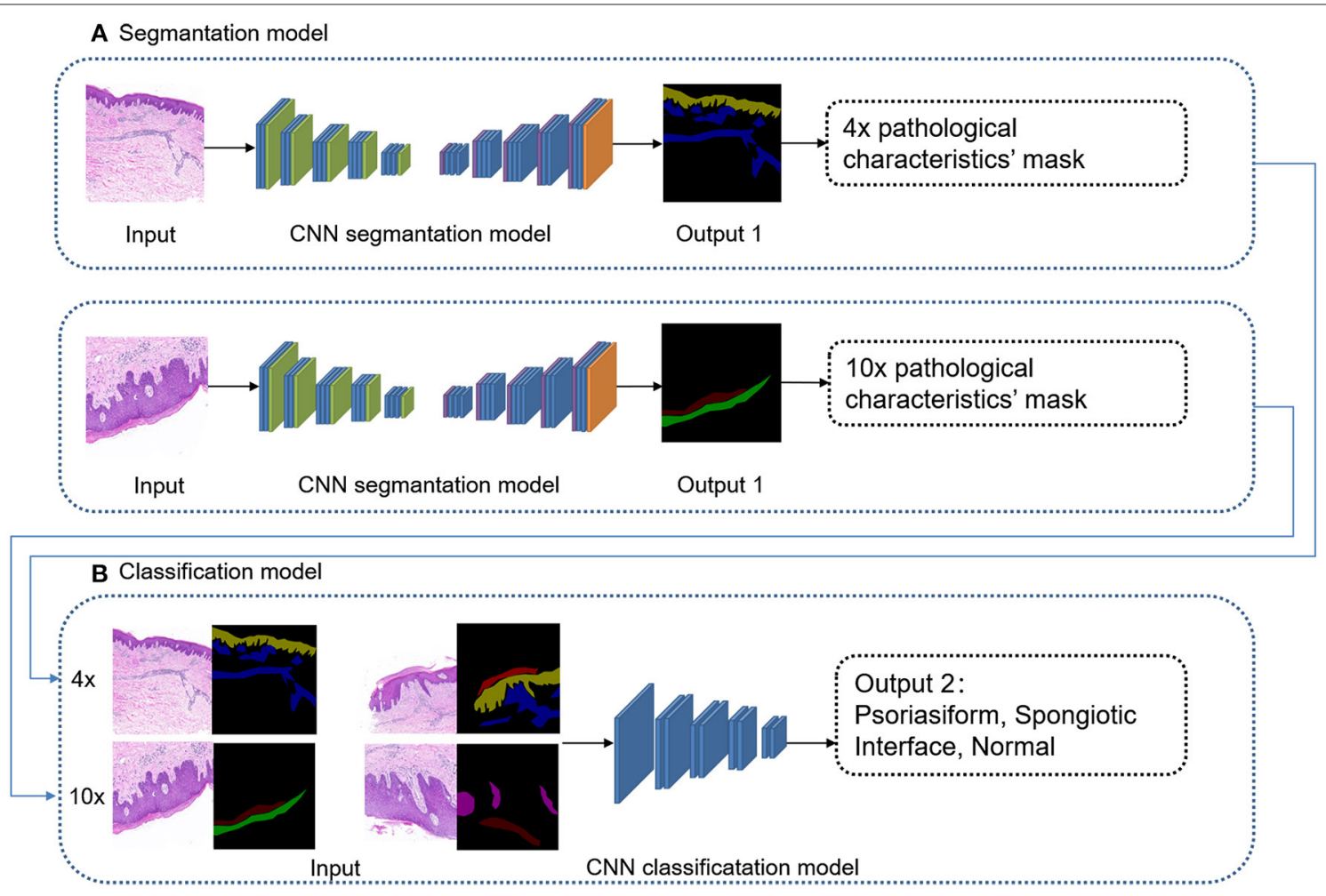

FIGURE 4 | Cascaded deep learning model of cascaded pathological characteristics recognition and subtype classification.

64.58\%(psoriasiform). Therefore, the cascaded recognition model for pathological characteristics was considerably effective in the subtype classification. Meanwhile, for the $4 \times$ images, the highest DICE coefficient was 0.8336 (acanthosis). For the $10 \times$ images, the highest DICE coefficient was 0.7262 (hypergranulosis). Table 5 and Figure 5 show the recognition results.

\section{DISCUSSION}

The principle of structure-pattern analysis is observing the pathological characteristics from low magnification to high magnification and identifying the subtypes of skin inflammations to provide key clues for a specific diagnosis. On the basis of this, a cascaded deep learning algorithm framework was proposed with cascaded pathological characteristics recognition and subtype classification. The pixel-by-pixel segmentation deep learning model "deeplabV3+" was employed to recognize pathological characteristics. Regions that contribute considerably to a diagnosis under a $4 \times$ magnification and $10 \times$ magnification were extracted through supervised learning for obtaining the pathological characteristics' masks of the original images and achieve recognition results with high consistency. The deep learning model "EfficientNet-b1" was 
employed for subtype classification. The mask output from the recognition model for pathological characteristics was combined with an image of the same section into a set of inputs, which jointly perform supervised learning to deliver accurate subtype classification.

TABLE 3 | Results of subtype classification of superficial perivascular dermatitis.

\begin{tabular}{ll}
\hline Classification model & $\begin{array}{l}\text { Classification } \\
\text { accuracy }(\%)\end{array}$ \\
\hline Efficientnet-B1 without recognition & 71.35 \\
Efficientnet-B1 cascaded U-Net & 79.88 \\
ResNet152 cascaded deeplab V3+ & 80.76 \\
Our model (Efficientnet-B1 & 85.24 \\
cascaded deeplab V3+) &
\end{tabular}

TABLE 4 | The different model parameter settings of training.

\begin{tabular}{|c|c|c|}
\hline Type of model & Model name & $\begin{array}{l}\text { Parameter settings of } \\
\text { training }\end{array}$ \\
\hline \multirow[t]{2}{*}{$\begin{array}{l}\text { The model of } \\
\text { recognition of } \\
\text { pathological } \\
\text { characteristics }\end{array}$} & Efficientnet-B1 & $\begin{array}{l}\mathrm{LR}=1 \mathrm{e}-4 \\
\text { batch_size }=8 \\
\mathrm{EPOCH}=201 \\
\text { Betas }=(0.9,0.999) \\
\text { eps }=1 \mathrm{e}-9\end{array}$ \\
\hline & U-Net & $\begin{array}{l}\mathrm{LR}=0.001 \\
\text { batch_size }=16 \\
\text { epochs }=100 \\
\text { img_scale }=0.5\end{array}$ \\
\hline \multirow[t]{2}{*}{$\begin{array}{l}\text { The model of subtype } \\
\text { classification }\end{array}$} & deeplab V3+ & $\begin{array}{l}\mathrm{LR}=0.01 \\
\mathrm{LR}=\mathrm{GAMMA}=0.1 \\
\text { BATCHES }=4 \\
\text { EPOCHS }=500\end{array}$ \\
\hline & ResNet152 & $\begin{array}{l}\text { learning_rate }=0.01 \\
\text { batch_size }=4 \\
\text { epoch }=500\end{array}$ \\
\hline
\end{tabular}

TABLE 5 | Results of pathological characteristics recognition.

\begin{tabular}{llc}
\hline Magnifications & Pathological characteristics region & DICE coefficient \\
\hline $4 \times$ & Hyperkeratosis & 0.6742 \\
& Acanthosis & 0.8336 \\
& Inflammatory cell infiltration & 0.5816 \\
& Lichenoid infiltration & 0.7729 \\
Parakeratosis & 0.1684 \\
Spongiosis & 0.3003 \\
Melanophages & 0.0121 \\
Hypergranulosis & 0.7262 \\
Hypogranulosis & 0.1621 \\
Blister & 0.1491 \\
Liquefaction degeneration of basal cells & 0.2239 \\
Angiectasis of dermal papillae & 0.4246 \\
Thinning of the suprapapillary epidermis & 0.4076
\end{tabular}

In this study, 13 different pathological characteristics were recognized which have a significant contribution to the subtype of inflammation. For dermatopathologists, identifying these pathological characteristics under different microscope multiples also brings important clues for the final diagnosis of the disease. The DICE coefficient ranges from 0 to 1 and the higher the DICE coefficient means the better the recognition effect. Thus, the acanthosis(DICE coefficient, $0.8,336$ ), lichenoid infiltration(DICE coefficient, 0.7,729), hypergranulosis(DICE coefficient, 0.7,262), hyperkeratosis(DICE coefficient, 0.6,742), were better recognized. These high recognition regions can make classification more accurate. As a result, lichenoid infiltration is the interface unique pathological characteristic, and its high recognition made the classification accuracy of interface subtype higher than psoriasiform and spongiotic. Altogether, the accuracy of subtype classification is $85.24 \%$, and the sensitivity and the specificity to be 67.46 and $89.09 \%$. This result is very close to the actual diagnostic accuracy of dermatopathologists in clinical work. As far as we know, the types of inflammatory skin diseases are complex. It is difficult to be specific dermatoses only by pathological diagnoses. The classification of subtypes and differential diagnosis given by pathologists according to the pathological characteristics have been the greatest help for clinicians. Our results have greatly reduced the artificial subjectivity of dermatopathologists. Compared with the previous recognition of benign or malignant tumors (14), the pathological characteristics involved in inflammation are dominated by cell infiltration and tissue structure changes, which involve multiple types of cells significantly complicated. In this study, the advantages of the pixel-level region segmentation model and the deep learning classification model were fully integrated, coupled with recognition of pathological characteristics, to render the subtype classification results more explanatory. The accuracy of subtype classification without recognition model was only $71.35 \%$, indicating that the performance of the cascaded deep learning model was considerably improved by the recognition of pathological characteristics.

This study was the first to focus on AI-aided pathological recognition of skin inflammation. Using fine-layered image labeling of small sample size, the structure-pattern analysis principle was simulated to simultaneously achieve automatic recognition of pathological characteristics and subtype classification. The color spaces of microscopic images were normalized into digitally scanned images. Both categories of images were recognizable. The three subtypes of inflammatory dermatoses selected in this study had diverse different pathological characteristics compared with normal tissues. Therefore, simplex subtype was not included because of the few pathological characteristics. As this study is the basic framework, the sample size in the future will be expanded to cover simplex subtype and the cascaded model will be generalized.

Although AI has great potential in aiding the recognition of pathological patterns of inflammatory dermatoses, the final diagnosis depends on the experience of the dermatopathologists and dermatologists. Our work would provide a valuable 


\section{A $4 \mathrm{X}$}

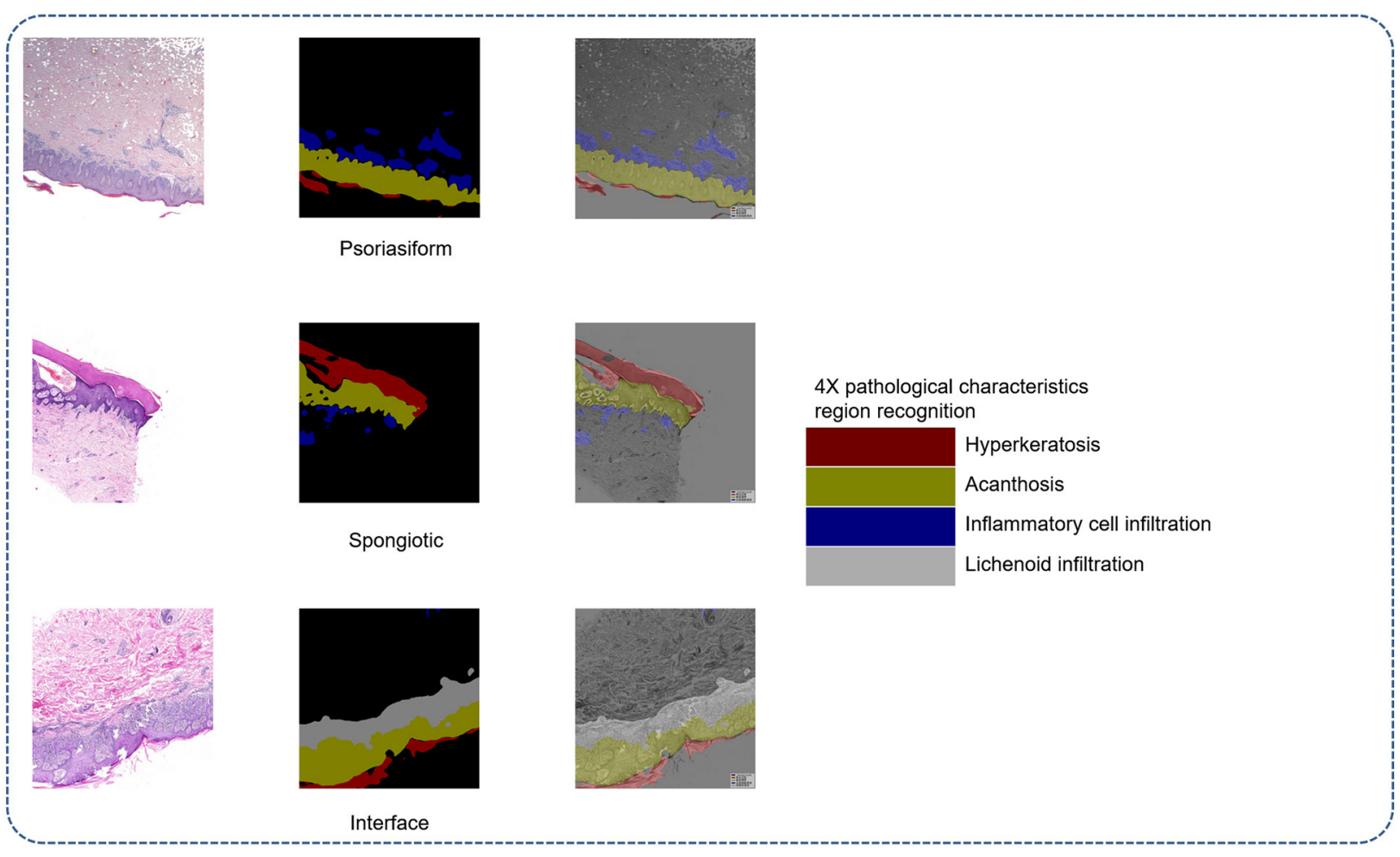

B $10 \mathrm{X}$

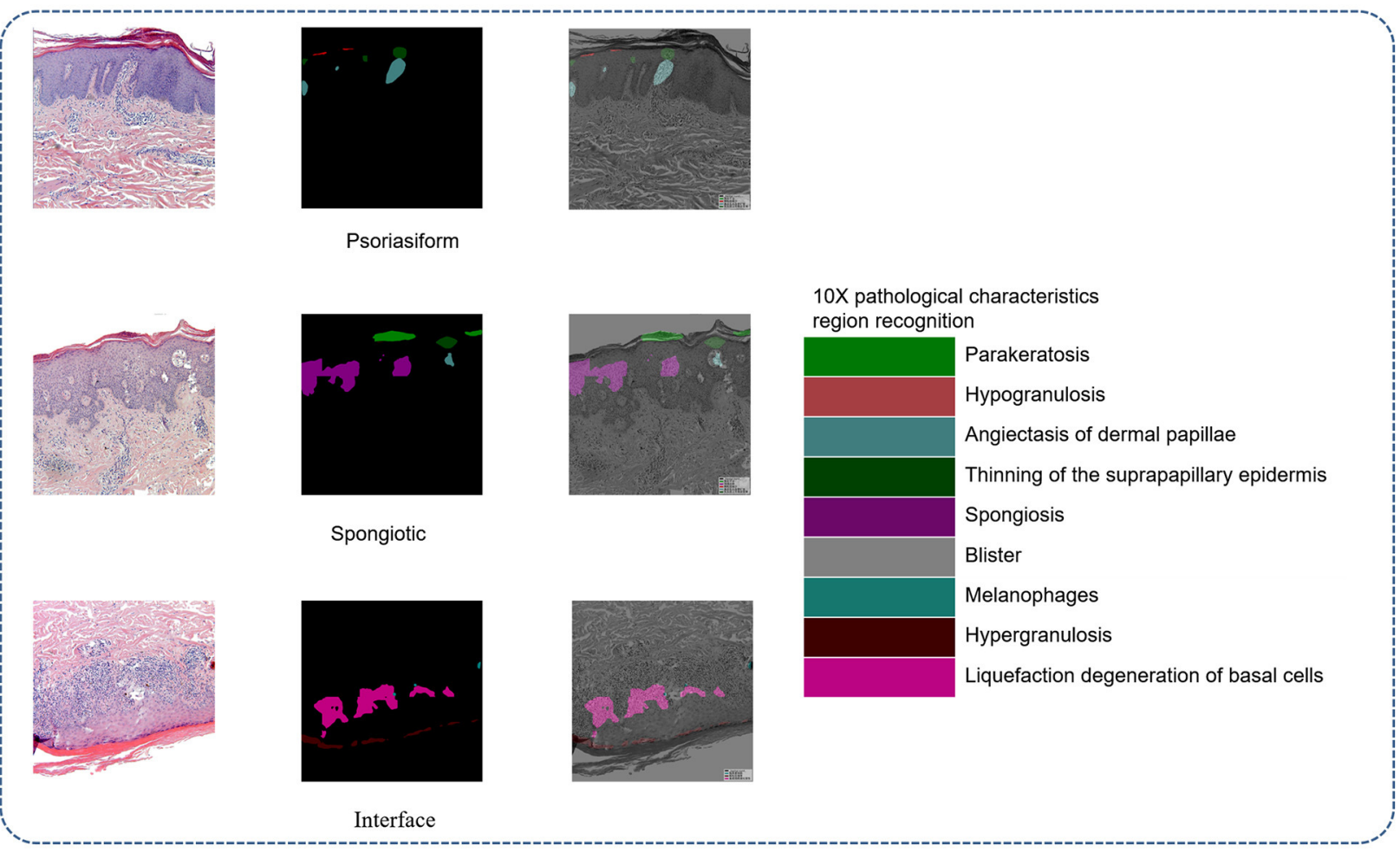

FIGURE 5 | Results of pathological characteristics region recognition. 
approach in allowing AI to facilitate dermatopathology better in the future.

\section{DATA AVAILABILITY STATEMENT}

The raw data supporting the conclusions of this article will be made available by the authors, without undue reservation.

\section{AUTHOR CONTRIBUTIONS}

YB and JZ carried out experiments and analyzed data. YF and JC conceived experiments and analyzed data. QZ and DL analyzed data. All the authors were involved in writing the paper and had final approval of the submitted and published versions.

\section{REFERENCES}

1. Ackerman $\mathrm{AB}$. Dermatopathology is for dermatologists and pathologists. Am J Derm Pathol. (1983) 5:107-8. doi: 10.1097/00000372-19830400000001

2. Zaharia D, Kanitakis J. Eruptive vellus hair cysts: report of a new case with immunohistochemical study and literature review. Dermatology. (2012) 224:15-9. doi: 10.1159/000337209

3. Hekler A, Utikal JS, Enk AH, Berking C, Klode J, Schadendorf D, et al. Pathologist-level classification of histopathological melanoma images with deep neural networks. Eur J Cancer. (2019) 115:79-83. doi: 10.1016/j.ejca.2019.04.021

4. Jiang YQ, Xiong JH, Li HY, Yang XH, Yu WT, Gao M, et al. Recognizing basal cell carcinoma on smartphone-captured digital histopathology images with a Deep Neural Network. $\mathrm{Br} J$ Dermatol. (2020) 182:754-62. doi: 10.1111/bjd.18026

5. Chen LC, Papandreou G, Kokkinos I, Murphy K, Yuille AL. DeepLab: semantic image segmentation with deep convolutional nets, atrous convolution, and fully connected CRFs. IEEE T-PAMI. (2018) 40:834-8. doi: 10.1109/TPAMI.2017.26 99184

6. Cruz-Roa AA, Arevalo Ovalle JE, Madabhushi A, González Osorio FA. A deep-learning architecture for image representation, visual interpretability and automated basal-cell carcinoma cancer detection. Intervention. (2013) 16:403-10. doi: 10.1007/978-3-642-40763-5_50

7. Liersch J, Von Köckritz A, Schaller J. Dermatopathology 101: part 1 inflammatory skin diseases. JDDG J Dtsch Dermatol Ges. (2017) 15:930. doi: $10.1111 /$ ddg. 13176

8. Polesie S, McKee PH, Gardner JM, Gillstedt M, Siarov J, Neittaanmäki N, et al. Attitudes toward artificial intelligence within dermatopathology: an international online survey. Front Med. (2020) 7:591952. doi: 10.3389/fmed.2020.591952

9. Esteva A, Kuprel B, Novoa RA, Ko J, Swetter SM, Blau HM, et al. Dermatologist-level classification of skin cancer with deep

\section{FUNDING}

This work is supported by Natural Science Foundation of Beijing 7202177 and Beijing Nova Program of Science and Technology Z191100001119053.

\section{ACKNOWLEDGMENTS}

We would like to thank Beijing hospital for providing tissues for study purposes.

\section{SUPPLEMENTARY MATERIAL}

The Supplementary Material for this article can be found online at: https://www.frontiersin.org/articles/10.3389/fmed. 2021.696305/full\#supplementary-material

neural networks. Nature. (2017) 542:115-8. doi: 10.1038/nature 21056

10. Olsen TG, Jackson BH, Feeser TA, Kent MN, Moad JC, Krishnamurthy $\mathrm{S}$, et al. Diagnostic performance of deep learning algorithms applied to three common diagnoses in dermatopathology. J Pathol Inform. (2018) 9:32. doi: 10.4103/jpi.jpi_31_18

11. Achi HE, Belousova T, Chen L, Wahed A, Wang I, Hu Z, et al. Automated diagnosis of lymphoma with digital pathology images using deep learning. Ann Clin Lab Sci. (2019) 49:153-160. Available online at: http://arxiv.org/abs/ 1811.02668

12. Smith EH, Chan MP. Inflammatory dermatopathology for general surgical pathologists. Clin Lab Med. (2017) 37:673-96. doi: 10.1016/j.cll.2017.05.008

13. Sengupta S, Jayaram V, Curless B, Seitz SM, Kemelmacher-Shlizerman I. Background matting: the world is your green screen. In: Proceedings of the IEEE/CVF Conference on Computer Vision and Pattern Recognition (CVPR). Seattle, WA: IEEE (2020) p. 2288-2297. doi: 10.1109/CVPR42600.2020.00236

14. Gomolin A, Netchiporouk E, Gniadecki R, Litvinov IV. Artificial intelligence applications in dermatology: where do we stand? Front Med. (2020) 7:100. doi: 10.3389/fmed.2020.00100

Conflict of Interest: JZ and DL were employed by company Beijing BeYes Technology Co. Ltd.

The remaining authors declare that the research was conducted in the absence of any commercial or financial relationships that could be construed as a potential conflict of interest.

Copyright (c) 2021 Bao, Zhang, Zhang, Chang, Lu and Fu. This is an open-access article distributed under the terms of the Creative Commons Attribution License (CC BY). The use, distribution or reproduction in other forums is permitted, provided the original author(s) and the copyright owner(s) are credited and that the original publication in this journal is cited, in accordance with accepted academic practice. No use, distribution or reproduction is permitted which does not comply with these terms. 